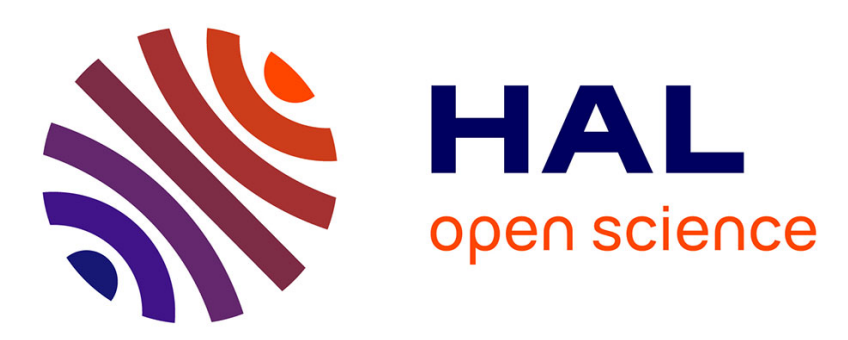

\title{
Adsorption and strain: The CO2-induced swelling of coal
} Matthieu Vandamme, Laurent Brochard, Brice Lecampion, Olivier Coussy

\section{To cite this version:}

Matthieu Vandamme, Laurent Brochard, Brice Lecampion, Olivier Coussy. Adsorption and strain: The CO2-induced swelling of coal. Journal of the Mechanics and Physics of Solids, 2010, 58 (10), pp.1489-1505. 10.1016/j.jmps.2010.07.014 . hal-00542308

\section{HAL Id: hal-00542308 https://hal.science/hal-00542308}

Submitted on 2 Dec 2010

HAL is a multi-disciplinary open access archive for the deposit and dissemination of scientific research documents, whether they are published or not. The documents may come from teaching and research institutions in France or abroad, or from public or private research centers.
L'archive ouverte pluridisciplinaire HAL, est destinée au dépôt et à la diffusion de documents scientifiques de niveau recherche, publiés ou non, émanant des établissements d'enseignement et de recherche français ou étrangers, des laboratoires publics ou privés. 


\section{Adsorption and Strain: The $\mathrm{CO}_{2}$-Induced Swelling of Coal}

M. Vandamme $^{1}$, L. Brochard ${ }^{2}$, B. Lecampion ${ }^{3}$, (the late) O. Coussy ${ }^{4 *}$

(1) Université Paris-Est, Laboratoire Navier (ENPC/LCPC/CNRS) 6-8 Av. B. Pascal, 77420 Champs-sur-Marne, France Corresponding author Phone: +331641537 04; Fax: +33164153741 E-mail address: matthieu.vandamme@enpc.fr

(2) Université Paris-Est, Laboratoire Navier (ENPC/LCPC/CNRS) 6-8 Av. B. Pascal, 77420 Champs-sur-Marne, France

(3) Schlumberger Carbon Services, 1 rue Henri Becquerel, 92140 Clamart, France

(4) Université Paris-Est, Laboratoire Navier (ENPC/LCPC/CNRS) 6-8 Av. B. Pascal, 77420 Champs-sur-Marne, France July 8, 2010

*The final version of the manuscript was prepared after the unfortunate passing of Professor Coussy 


\begin{abstract}
Enhanced coal bed methane recovery (ECBM) consists in injecting carbon dioxide in coal bed methane reservoirs in order to facilitate the recovery of the methane. The injected carbon dioxide gets adsorbed at the surface of the coal pores, which causes the coal to swell. This swelling in confined conditions leads to a closure of the coal reservoir cleat system, which hinders further injection. In this work we provide a comprehensive framework to calculate the macroscopic strains induced by adsorption in a porous medium from the molecular level. Using a thermodynamic approach we extend the realm of poromechanics to surface energy and surface stress. We then focus on how the surface stress is modified by adsorption and on how to estimate adsorption behavior with molecular simulations. The developed framework is here applied to the specific case of the swelling of $\mathrm{CO}_{2}$-injected coal, although it is relevant to any problem in which adsorption in a porous medium causes strains.
\end{abstract}

Keywords: Porous material (B), constitutive behavior (B), adsorption, molecular simulations

Methane production from unminable coal seams -denoted as Coal Bed Methane (CBM)- has amounted in 2008 to about $10 \%$ of the total natural gas production in the United States, while large commercial projects also operate in Australia, China and India (Jenkins and Boyer, 2008). The general characteristics of these reservoirs vary widely with depth ranging from 0.3 to $1.3 \mathrm{~km}$ and bed thickness between 1 to 30 meters. Coal reservoirs typically have low permeability, high compressibility, and production is mostly driven by gas desorption. Gas is stored mostly in the coal material (to which we simply refer to as 'coal') while most, if not all, of the permeability comes from the set of small natural fractures (often denoted cleats) typically present at a scale of a few centimeters (see figure 1). Such a 'cleats driven' permeability is highly stress dependent. Due to the higher adsorption properties of carbon dioxide compared to methane in coal, the technique of injecting $\mathrm{CO}_{2}$ in coal beds enhances methane production while possibly providing a way to store $\mathrm{CO}_{2}$. It could therefore ultimately lower the level of $\mathrm{CO}_{2}$ emissions associated with consumption of methane produced from coal beds (White et al., 2005). Such a process known as enhanced coal bed methane recovery (ECBM) dates back from the beginning of the eighties; its application has been largely correlated with a higher gas price period during which CBM was more beneficial economically. An interest within the scope of $\mathrm{CO}_{2}$ storage 
has also arisen with research projects in Europe (Van Bergen et al., 2009) and China (Wong et al., 2007), field applications in the United States (e.g., the Allison project) and field application proposals in Australia (e.g., the Fairview project).

One of the most important aspects of ECBM performance is linked with the so-called differential swelling induced by the preferential adsorption properties of $\mathrm{CO}_{2}$ with respect to $\mathrm{CH}_{4}$ in coal (Busch et al., 2003). Such a $\mathrm{CO}_{2}$ induced swelling of coal leads to an initial decrease in permeability (see the early stage of $\mathrm{CO}_{2}$ injection on figure 2a) via the closing of the cleat system, which hinders further injection of $\mathrm{CO}_{2}$. After this initial decrease permeability increases back (see the increase of injectivity in the late stages of $\mathrm{CO}_{2}$ injection on figure 2a), a phenomenon known as the "permeability rebound' and attributed to a reopening of the cleats due to an increase over time of the fluid pressure in the cleat system. The prediction of such permeability variations is of great interest in field applications in order to design wells completion and plan mitigation methods to restore injectivity (e.g., hydraulic fracturing of the injection wells). A first step in that direction is to consistently understand and describe the $\mathrm{CO}_{2}$ induced swelling of the coal material: Our aim in this paper.

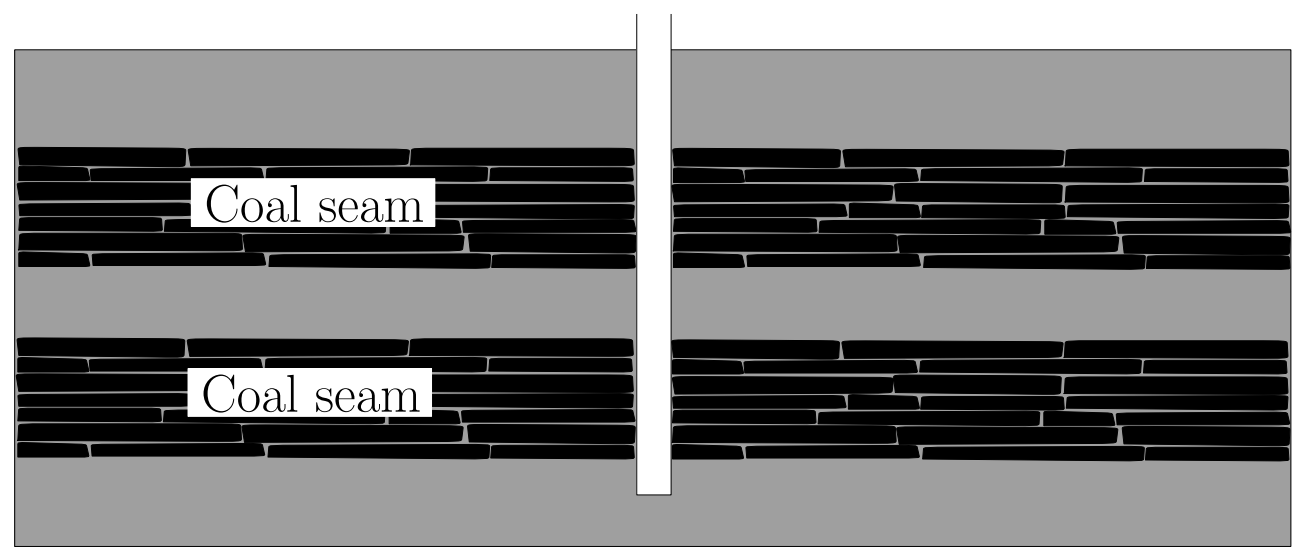

Figure 1: CBM well. A typical vertical CBM well goes through several coal seams. A coal seam is made of coal material (in black) naturally fractured. Fractures are called 'cleats.'

Coal is by nature porous with pores ranging from tenths of micron to tenths of nanometer (Gan et al., 1972). One can attempt to apply the usual tools of poromechanics and assess whether those tools enable to capture the 
a)

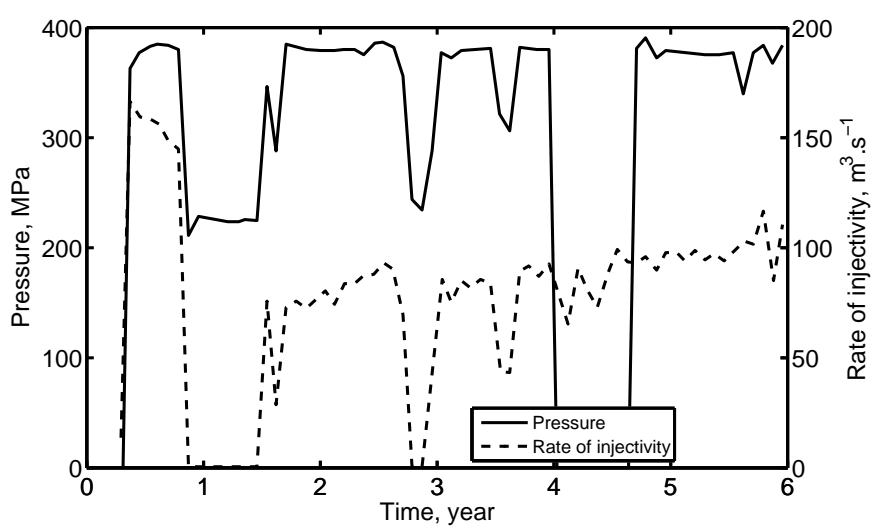

b)

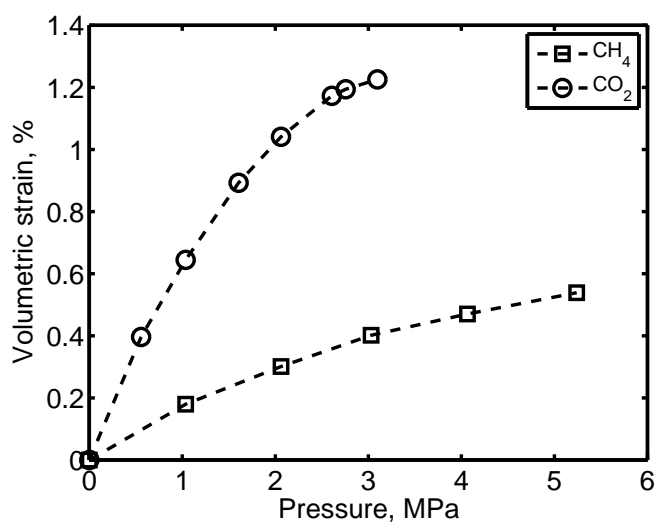

Figure 2: Experimental data on injected coal: (a) Decline in injectivity over time in a $\mathrm{CO}_{2}$-injected coal bed at the Allison unit in the San Juan basin (adapted from Reeves (2004)) and (b) volumetric strain versus pore pressure in a $\mathrm{CO}_{2}$-injected coal and in a $\mathrm{CH}_{4}$-injected coal (adapted from Levine (1996)). 
coal differential swelling consecutive to an injection of carbon dioxide. For an isotropic porous medium the equations of poroelasticity link the mean confining stress $\sigma$, the confining deviatoric stresses $s_{i j}$, and the pore pressure $p$ to the volumetric dilation $\epsilon$, the deviatoric strains $e_{i j}$ and the change $\varphi$ of porosity $\left(\varphi=\phi-\phi_{0}\right.$, where $\phi$ is the actual porosity and $\phi_{0}$ the porosity in the state of reference) through the bulk modulus $K$, the shear modulus $G$, the Biot coefficient $b$, and the Biot modulus $N$ (Coussy, 2004):

$$
\begin{aligned}
\sigma & =K \epsilon-b p \\
\varphi & =b \epsilon+p / N \\
s_{i j} & =2 G e_{i j}
\end{aligned}
$$

In the above equations $K$ and $G$ are the elastic moduli of the coal material as a whole, i.e., made of the coal solid matrix (with its own bulk modulus $\left.k_{s} \neq K\right)$ and of its pore space.

Figure $2 \mathrm{~b}$ readily shows the differential swelling of coal: At a given pressure of the pore fluid the swelling during an unjacketed experiment $(\sigma=-p)$ depends on the nature of the pore fluid. But this experimental observation can not be captured by usual poromechanics since equation (1) links in a unique manner mean stress, volumetric dilation, and pore pressure, independently of the nature of the pore fluid. This discrepancy stems from the main assumptions on which equations (1-3) are derived. As will be discussed later on, this derivation is performed by assuming that free energy can be stored into the porous medium in only two ways: Elastic deformation of the solid matrix and addition of pore fluid. It thus disregards a third way of storing energy: At the interface between the solid matrix and the pore space. The effect of surface energy is often disregarded in poromechanics. It is however needed in order to properly describe the $\mathrm{CO}_{2}$-induced swelling of coal, which is due to the adsorption of fluid at the interface between solid matrix and pore space (Bangham and Maggs, 1944; Harpalani and Schraufnagel, 1990; Yates, 1954). How adsorption leads to strain has been studied by several, but the results remain mostly based on specific microstructures (e.g., Scherer (1986) or Kowalczyk et al. (2008)) and/or on specific sorption strain models (e.g., Pan and Connell (2007) or Palmer and Mansoori (1998) for coal and its implication on permeability changes). The work presented herein aims at proposing a more general framework through which to compute strains caused by adsorption. 
In a first section the realm of poromechanics is extended in order to take into account surface energy, which will enable to compute the strain caused by a given surface stress for any pore structure. This extension is achieved by using a thermodynamic approach. We also make the connection with a micromechanical approach available in the literature. In a second section we focus on how adsorption modifies the surface stress. A third section deals with the estimation of the adsorption behavior; an approach based on molecular simulations is notably presented. The results are eventually compared with laboratory data of $\mathrm{CO}_{2}$ and $\mathrm{CH}_{4}$ injection in coal.

\section{Surface energy and poromechanics}

This section is devoted to the extension of poromechanics to surface energy effects. We consider a porous solid saturated with a fluid. We aim at quantifying the strain induced by surface effects.

\subsection{Extension of poromechanics to surface effects}

We consider a unit volume of saturated porous medium. The medium has a porosity $\phi$ and is subject to the macroscopic stress tensor $\sigma_{i j}$. The Helmholtz free energy of this porous medium per unit volume is noted $f$, that of the fluid is noted $f_{l}$. By introducing $f_{s}=f-f_{l}$ one can derive the following energy balance at equilibrium (Coussy, 2004) (throughout the text an implicit sum is to be carried out over repeated indices):

$$
\mathrm{d} f_{s}=\mathrm{d} f-\mathrm{d} f_{l}=\sigma \mathrm{d} \epsilon+\sum_{i, j} s_{i j} \mathrm{~d} e_{i j}+p \mathrm{~d} \varphi
$$

From the definition of $f_{s}$ (i.e., $f_{s}=f-f_{l}$ ) the above equation is identified as the energy balance at equilibrium for the system made of the porous medium without its fluids. Work can be provided to this system by the action of stresses, either volumetric (' $\sigma \mathrm{d} \epsilon$ ') or deviatoric ( ${ }^{i j} \mathrm{~d} e_{i j}$ '), as is the case for ordinary solids. But work can also be provided to this system by the action of pressure against the pore walls (' $p \mathrm{~d} \varphi$ '). From equation (4) the state equations of poroelasticity can then be derived:

$$
\sigma=\frac{\partial f_{s}}{\partial \epsilon}, s_{i j}=\frac{\partial f_{s}}{\partial e_{i j}}, \text { and } p=\frac{\partial f_{s}}{\partial \varphi}
$$


If the system made of the porous medium without its fluids is identified as being the solid matrix, one can then perform the following derivation. From equation (4) $f_{s}$ is a function of strains and porosity, i.e., $f_{s}=\psi_{s}\left(\epsilon, e_{i j}, \varphi\right)$. Within the frame of linear elasticity $f_{s}$ must be a quadratic function of its arguments. If the solid matrix is isotropic, deviatoric strains are coupled with neither change of porosity nor volumetric strain. $f_{s}$ can then be written as, e.g.,:

$$
f_{s}=\psi_{s}\left(\epsilon, e_{i j}, \varphi\right)=\frac{1}{2}\left(K+b^{2} N\right) \epsilon^{2}-b N \epsilon \varphi+\frac{N}{2} \varphi^{2}+G \sum_{i, j} e_{i j} e_{j i}
$$

and equation (5) reduces to the constitutive equations (1-3) in which no effect of surface energy is included.

If surface energy is to be included, one has to recognize that the system made of the porous medium without its fluids does not only contain the solid matrix but also the solid-fluid interface (i.e., the interface between the solid matrix and the pore space). The free energy $f_{s}$ has therefore to be split into the energy stored elastically within the solid matrix (the free energy $\psi_{s}$ introduced in equation (6)) and into the energy $u$ stored at the solid-fluid interface:

$$
f_{s}=\psi_{s}\left(\epsilon, e_{i j}, \varphi\right)+u\left(\epsilon, e_{i j}, \varphi\right)
$$

where $f_{s}, \psi_{s}$ and $u$ are expressed per unit volume of porous medium in the configuration of reference.

Energy can be stored at an interface as well as energy can be stored in a membrane being stretched. Therefore, analogous to a membrane force, a force opposes an elastic deformation of the interface. We call this force the surface stress ${ }^{1}$. As defined by Kramer and Weissmüller (2007), the "surface stress $[. .$.$] measures the forces which oppose an elastic deformation of the$ surface, changing the interatomic distance at constant number of atoms." Therefore the energy balance for the interface at equilibrium is given by:

$$
\mathrm{d} u=\tilde{\sigma}^{s} \mathrm{~d} s
$$

\footnotetext{
${ }^{1}$ The surface stress $\tilde{\sigma}^{s}$ had not to be confused with the more familiar notion of surface tension. The difference between surface tension (which we later on name 'interface energy' to avoid any confusion) and surface stress is explained in great detail in section 2 .
} 
where $\tilde{\sigma}^{s}$ is the surface stress and $s$ is the actual area of the pore walls per unit volume of porous medium in the configuration of reference. Because of the assumed linearity of the solid matrix a small variation $\mathrm{d} s$ can be expressed for infinitesimal transformations as $\mathrm{d} s=\partial s /\left.\partial \epsilon\right|_{\varphi=0 ; e_{i j}=0} \mathrm{~d} \epsilon+$ $\partial s /\left.\partial e_{i j}\right|_{\epsilon=0 ; \varphi=0} \mathrm{~d} e_{i j}+\partial s /\left.\partial \varphi\right|_{\epsilon=0 ; e_{i j}=0} \mathrm{~d} \varphi$. In first order the area of a surface remains unchanged by deviatoric strains and $\partial s /\left.\partial e_{i j}\right|_{\epsilon=0 ; \varphi=0}=0$. Equations (7) and (8) combined with equation (4) provide the following energy balance:

$$
\mathrm{d} \psi_{s}=\left(\sigma-\sigma_{a}\right) \mathrm{d} \epsilon+\sum_{i, j} s_{i j} \mathrm{~d} e_{i j}+\left(p-p_{a}\right) \mathrm{d} \varphi
$$

where the stress $\sigma_{a}$ and the pressure $p_{a}$ related to adsorption are:

$$
\sigma_{a}=\left.\tilde{\sigma}^{s} \frac{\partial s}{\partial \epsilon}\right|_{\varphi=0} \text { and } p_{a}=\left.\tilde{\sigma}^{s} \frac{\partial s}{\partial \varphi}\right|_{\epsilon=0}
$$

If we restrain ourselves to linear poroelasticity the potential $\psi_{s}$ is the quadratic function given by equation (6), and equation (9) yields:

$$
\begin{aligned}
\sigma-\sigma_{a} & =\frac{\partial \psi_{s}}{\partial \epsilon}=\left(K+b^{2} N\right) \epsilon-b N \varphi \\
p-p_{a} & =\frac{\partial \psi_{s}}{\partial \varphi}=-b N \epsilon+N \varphi \\
s_{i j} & =\frac{\partial \psi_{s}}{\partial e_{i j}}=2 G e_{i j}
\end{aligned}
$$

from what follow the constitutive equations:

$$
\begin{aligned}
\sigma-\sigma_{a} & =K \epsilon-b\left(p-p_{a}\right) \\
\varphi & =b \epsilon+\left(p-p_{a}\right) / N \\
s_{i j} & =2 G e_{i j}
\end{aligned}
$$

With regard to the standard constitutive equations of isotropic poroelasticity (1-3), equations (14-16) show that a pre-stress $\sigma_{a}$ and an initial pore pressure $p_{a}$ have to be applied against the effects induced by the surface stress $\tilde{\sigma}^{s}$ in order to prevent any deformation and any porosity change with respect to the reference configuration. 
Conversely, if we let $\sigma=p=0$ but $\tilde{\sigma}^{s} \neq 0$ (and therefore $\sigma_{a} \neq 0$ and $p_{a} \neq 0$ ), we get a non zero strain $\epsilon_{a} \neq 0$ and a non zero change of porosity $\varphi_{a} \neq 0$ :

$$
\begin{aligned}
\epsilon_{a} & =-\left(\sigma_{a}+b p_{a}\right) / K=-\alpha_{\epsilon} \tilde{\sigma}^{s} \\
\varphi_{a} & =b \epsilon_{a}-p_{a} / N=-\alpha_{\varphi} \tilde{\sigma}^{s}
\end{aligned}
$$

where $\alpha_{\epsilon}$ and $\alpha_{\varphi}$ are constant material parameters. With the help of equation (10) and the use of a Legendre-Fenchel transform those material parameters can be expressed as:

$$
\begin{aligned}
& \alpha_{\epsilon}=\frac{1}{K}\left(\left.\frac{\partial s}{\partial \epsilon}\right|_{\varphi=0}+\left.b \frac{\partial s}{\partial \varphi}\right|_{\epsilon=0}\right)=\left.\frac{1}{K} \frac{\partial s}{\partial \epsilon}\right|_{p=0} \\
& \alpha_{\varphi}=\left.\frac{b}{K} \frac{\partial s}{\partial \epsilon}\right|_{\varphi=0}+\left.\frac{1}{N} \frac{\partial s}{\partial \varphi}\right|_{\epsilon=0}=\left.\left(\frac{b^{2}}{K}+\frac{1}{N}\right) \frac{\partial s}{\partial \varphi}\right|_{\sigma=0}
\end{aligned}
$$

and the set of constitutive equations (14-16) can thus be rewritten as:

$$
\begin{aligned}
\sigma & =K\left(\epsilon-\epsilon_{a}\right)-b p \\
\varphi-\varphi_{a} & =b\left(\epsilon-\epsilon_{a}\right)+p / N \\
s_{i j} & =2 G e_{i j}
\end{aligned}
$$

Up to the first order for infinitesimal transformations a change in the surface stress $\tilde{\sigma}^{s}$ only affects $\epsilon_{a}$ and $\varphi_{a}$.

The parameters $\alpha_{\epsilon}$ and $\alpha_{\varphi}$ needed in order to compute $\epsilon_{a}$ and $\varphi_{a}$ are a function of the mechanical properties of the solid matrix and of the geometrical arrangement of the solid matrix in the volume of the porous medium. The evaluation of those parameters and thus of the strain induced by surface effects is theoretically possible for any porous medium as long as the microstructure of the medium and the mechanical properties of the matrix are fully known. Equations (19) and (20) show that these parameters can also be measured directly at the macroscopic scale: When varying the confining pressure while keeping the pore pressure constant, $\partial s /\left.\partial \epsilon\right|_{p=0}$ is the change of the area of the pore walls (per unit volume of porous medium) with respect to a change of volumetric strain; and when varying the pore pressure while 
keeping the confining stress constant, $\partial s /\left.\partial \varphi\right|_{\sigma=0}$ is the change of the area of the pore walls (per unit volume of porous medium) with respect to a change of porosity.

In the next section the above derivations are applied to a porous medium with a simple microstructure. Their application to a porous medium with a general microstructure is then compared with a micromechanical approach by Dormieux et al. (2006).

\subsection{Application to a porous medium with a simple mi- crostructure}

a)

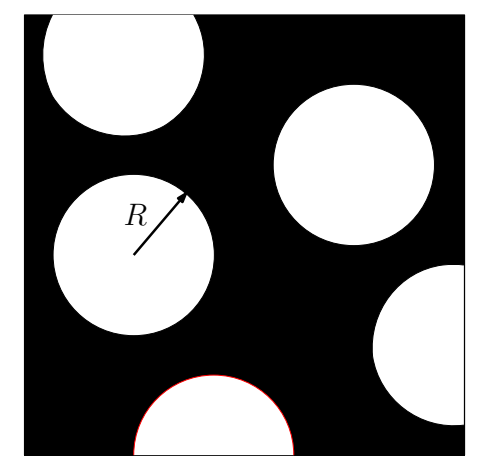

b)

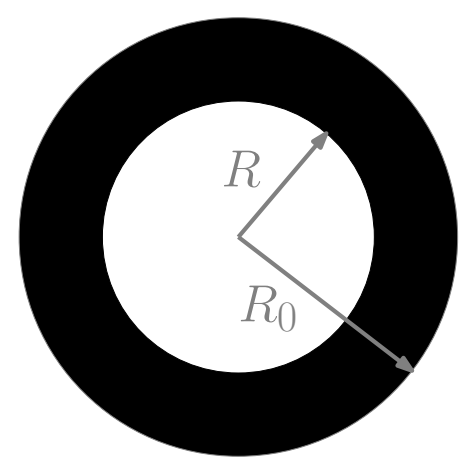

Figure 3: (a) Porous medium with mono-sized spherical pores; and (b) its approximate morphology: Spherical pore surrounded with solid.

In this section we consider an ideal microstructure: A porous medium with mono-sized spherical pores (figure 3a). For this simple microstructure the volumetric strains due to surface effects can be fully calculated with the derivations performed in section 1.1. The morphology of such a medium can be reasonably well captured by considering each pore as surrounded by a crust of solid (figure 3b). The volumetric strain induced by surface energy effects for the porous medium will therefore be estimated by that for the approximate cell displayed in figure $3 \mathrm{~b}$.

The crust of solid of the approximate cell must have an outside radius $R_{0}$ that verifies:

$$
\phi=R^{3} / R_{0}^{3}
$$


in order for the porosity of the approximate cell to be equal to that of the initial porous medium here considered. Equation (19) shows that both $\partial s /\left.\partial \epsilon\right|_{\varphi=0}$ and $\partial s /\left.\partial \varphi\right|_{\epsilon=0}$ must be determined in order to calculate the strain $\epsilon_{a}$ induced by surface energy effects.

The quantity $\partial s /\left.\partial \epsilon\right|_{\varphi=0}$ links a small volumetric strain $\mathrm{d} \epsilon$ applied at constant porosity $(\varphi=0)$ with a small variation $\mathrm{d} s$ of the area of the interface per unit volume. Both porosity and surface per unit volume are Lagrangian variables, in the sense that they are to be calculated with respect to the volume of reference. The constraint of constant Lagrangian porosity $(\mathrm{d} \phi=0$ and therefore $\mathrm{d} R=0$ ) implies that for any volumetric strain applied to the cell the volume $4 \pi R^{3} / 3$ of the pore and the area $4 \pi R^{2}$ of the interface remain constant. From what we infer:

$$
\left.\frac{\partial s}{\partial \epsilon}\right|_{\varphi=0}=0
$$

The quantity $\partial s /\left.\partial \varphi\right|_{\epsilon=0}$ links a small variation of porosity $\mathrm{d} \varphi$ applied at constant volume $(\epsilon=0)$ with a small variation $\mathrm{d} s$ of the area of the interface per unit volume. The constraint of constant volume $\left(R_{0}\right.$ is constant) implies with equation (24) that $\mathrm{d} \varphi=3 R^{2} \mathrm{~d} R / R_{0}^{3}$. The area $s$ per unit volume is $s=4 \pi R^{2} /\left(4 \pi R_{0}^{3} / 3\right)=3 R^{2} / R_{0}^{3}$ by definition and the constraint of constant volume implies that $\mathrm{d} s=6 R \mathrm{~d} R / R_{0}^{3}$. A combination of those equations yields:

$$
\left.\frac{\partial s}{\partial \varphi}\right|_{\epsilon=0}=\frac{6 R \mathrm{~d} R}{R_{0}^{3}} / \frac{3 R^{2} \mathrm{~d} R}{R_{0}^{3}}=\frac{2}{R}
$$

which enables with equation (25) to calculate the material parameters $\alpha_{\epsilon}$ and $\alpha_{\varphi}$ :

$$
\alpha_{\epsilon}=2 b / K R ; \alpha_{\varphi}=2 / N R
$$

Therefore we can calculate the volumetric strain $\epsilon_{a}$ induced by surface effects for the cell displayed in figure $3 \mathrm{~b}$ and thus for a porous medium with spherical pores of radius $R$ :

$$
\epsilon_{a}=-\frac{\tilde{\sigma}^{s}}{K}\left(\left.\frac{\partial s}{\partial \epsilon}\right|_{\varphi=0}+\left.b \frac{\partial s}{\partial \varphi}\right|_{\epsilon=0}\right)=-\frac{2 \tilde{\sigma}^{s} b}{K R}
$$

In contrast, we can also consider another ideal structure: A granular material made of monosized spherical grains of radius $R$. The mean stress $p_{g}$ 
that prevails in the spherical grains is given by Young-Laplace's law (Laplace, 1843):

$$
p_{g}=\frac{2 \tilde{\sigma}^{s}}{R}
$$

If we now introduce the bulk modulus $k_{s}$ of the solid grains $\left(k_{s} \neq K\right)$, the volumetric srain of the granular medium is given by:

$$
\epsilon_{a}=-\frac{2 \tilde{\sigma}^{s}}{k_{s} R}
$$

A comparison of equation (28) with equation (30) shows that the order of magnitude of the contraction $\epsilon_{a}$ induced by surface energy effects strongly depends on the microstructure: If the porous medium is much softer than its solid phase (i.e., $K<<k_{s}$ ), the contraction can be much more significant if the porous medium is made of spherical voids (the contraction is then proportional to $1 / K$ ) than if the porous medium is made of solid grains (the contraction is then proportional to $\left.1 / k_{s}\right)$.

\subsection{Application to a porous medium with a general microstructure}

If we now consider a porous medium with a general microstructure, the strain induced by surface stress can theoretically be calculated: in order to do so, one needs to solve a poroelastic problem to calculate the material parameters $\partial s /\left.\partial \epsilon\right|_{p=0}$ and $\partial s /\left.\partial \varphi\right|_{\sigma=0}$, and then $\alpha_{\epsilon}$ and $\alpha_{\varphi}$.

There exists however an alternative way of calculating the volumetric strain induced by surface stress. Indeed the question of the link between surface stress and deformation has already been tackled by Dormieux et al. (2006) using a micromechanical approach distinct from the thermodynamical approach developed in this paper. This section is devoted to showing that both the micromechanical approach of Dormieux et al. and our thermodynamical approach provide equivalent results for the strain induced by surface effects.

Dormieux et al. start their micromechanical approach with choosing a representative elementary volume of the porous medium. This representative elementary volume has an initial volume $\Omega_{0}$ and is made of solid and pores separated by an interface $I_{s f}$. Surface effects are introduced as a discontinuity 
of the normal stress at the interface, the jump $\Delta \sigma_{n}$ in normal stress across the interface being:

$$
\Delta \sigma_{n}=\tilde{\sigma}^{s}\left(\underline{\underline{1}}_{T}: \underline{\underline{\operatorname{grad}}}(\underline{n})\right)
$$

where $\underline{\underline{1}}_{T}$ is the second-order unit tensor tangential to the solid surface. Considering that the surface stress creates a pre-stress in the material, and making use of the Levin's theorem, Dormieux et al. provide a general expression for the second-order strain tensor $\underline{\underline{\varepsilon_{a}}}$ induced by surface energy effects:

$$
\underline{\underline{\varepsilon_{a}}}=-\tilde{\sigma}^{s} \mathbb{S}^{h o m}:\left(\frac{1}{\Omega_{0}} \int_{I_{s f}} \underline{\underline{1}}_{T}: \mathbb{A}(x) d S\right)
$$

where $\mathbb{S}^{\text {hom }}$ is the fourth-order compliance tensor, and $\mathbb{A}(x)$ is the fourthorder so-called strain localization tensor. This strain localization tensor, well known in micromechanics, links the microscopic strain tensor $\underline{\underline{\varepsilon}}^{\text {micro }}(x)$ at the scale of the microstructure with the macroscopic strain tensor $\underline{\underline{\varepsilon}}$ at the scale of the porous medium:

$$
\underline{\underline{\varepsilon}}^{\text {micro }}(x)=\mathbb{A}(x): \underline{\underline{\varepsilon}}
$$

We note $S_{s f}$ the area of the interface within the volume $\Omega_{0}$. A small strain $\underline{\underline{\mathrm{d} \varepsilon}}$ is applied to the volume. By definition the local strain $\underline{\underline{\mathrm{d} \varepsilon}}^{\text {micro }}(x)$ is $\underline{\underline{\mathrm{d}}}^{\text {micro }}(x)=\mathbb{A}(x): \underline{\underline{\mathrm{d} \varepsilon}}$.

By definition again the change of area $\mathrm{d} S_{s f}$ of the interface due to the

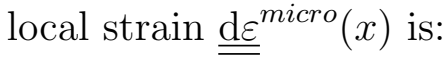

$$
\begin{aligned}
\mathrm{d} S_{s f}=\int_{I_{s f}} \underline{\underline{1}}_{T}: \underline{\underline{\mathrm{d} \varepsilon}}^{\text {micro }}(x) \mathrm{d} S & =\int_{I_{s f}} \underline{\underline{1}}_{T}: \mathbb{A}(x): \underline{\underline{\mathrm{d} \varepsilon}} \mathrm{d} S \\
& =\left(\int_{I_{s f}} \underline{1}_{T}: \mathbb{A}(x) \mathrm{d} S\right): \underline{\underline{\mathrm{d} \varepsilon}}
\end{aligned}
$$

which, since $s=S_{s f} / \Omega_{0}$, can be rewritten as:

$$
\left.\frac{\partial s}{\partial \underline{\underline{\varepsilon}}}\right|_{p=0}=\frac{1}{\Omega_{0}}\left(\int_{I_{s f}} \underline{1}_{T}: \mathbb{A}(x) d S\right)
$$

from what follows, with the help of equation (32): 


$$
\underline{\underline{\varepsilon_{a}}}=-\tilde{\sigma}^{s} \mathbb{S}^{h o m}:\left.\frac{\partial s}{\partial \underline{\underline{\varepsilon}}}\right|_{p=0}
$$

By assuming that the material is isotropic at the scale of the porous medium the macroscopic compliance tensor can be expressed as $\mathbb{S}^{\text {hom }}=$ $(1 / 3 K) \mathbb{J}+(1 / 2 G) \mathbb{K}$ where $\mathbb{K}$ and $\mathbb{J}$ are the volumetric and deviatoric parts of the fourth-order unity tensor, respectively; and where $K$ and $G$ are the macroscopic bulk and shear moduli of the porous medium, respectively. In first order, since the porous medium is assumed isotropic, the area $S_{s f}$ of the interface is not modified by deviatoric strains so that $\partial s /\left.\partial \underline{\underline{\varepsilon}}\right|_{p=0}=$ $\partial s /\left.\partial \epsilon\right|_{p=0} \mathbb{K}$. Equation (37) reduces to:

$$
\epsilon_{a}=-\left.\frac{\tilde{\sigma}^{s}}{K} \frac{\partial s}{\partial \epsilon}\right|_{p=0}
$$

which we also obtained with our thermodynamical approach by combining equations (17) and (19): The micromechanical approach and the approach developed in section 1.1 provide equivalent results.

For materials whose inhomogeneities are ellipsoidal (which accounts for a large variety of materials), based on the Eshelby inclusion problem, micromechanics can provide estimates of the strain localization tensor $\mathbb{A}(x)$, which makes it very convenient to use equation (32). On the other hand, our thermodynamical approach provides the physical meaning of equation (32).

For the simple porous medium considered in section 1.2 with mono-sized spherical pores, assuming that the mechanical interaction between pores is negligible, the strain localization tensor $\mathbb{A}(x)$ can be estimated by a constant fourth-order tensor $\mathbb{A}^{p}$ which verifies (Dormieux et al., 2006):

$$
\underline{\underline{1}}: \mathbb{A}^{p}=\frac{b}{\phi} \underline{\underline{1}}
$$

where $\phi$ is the porosity of the medium and $b$ its Biot coefficient. Noting that $\int_{I_{s f} \underline{\underline{1}}_{T}} d S=8 \pi R^{2} \underline{\underline{1}}$ and with the help of the above equation, equation (32) becomes:

$$
\underline{\underline{\varepsilon_{a}}}=-\tilde{\sigma}^{s} \frac{8 \pi R^{2} b}{\Omega_{0} \phi} \mathbb{S}^{h o m}: \underline{\underline{1}}=-\tilde{\sigma}^{s} \frac{8 \pi R^{2} b}{\Omega_{0} \phi} \frac{1}{9 K} \underline{\underline{1}}
$$

from which, since $\Omega_{0} \phi=4 \pi R^{3} / 3$, the volumetric strain induced by a surface stress is estimated to: 


$$
\epsilon_{a}=\operatorname{tr}\left(\underline{\underline{\varepsilon_{a}}}\right)=-\frac{2 \tilde{\sigma}^{s} b}{K R}
$$

A comparison of the above equation with equation (28) readily shows that, as expected, the micromechanical approach and our thermodynamical approach yield the same results on the simple porous medium considered.

As long as the microstructure is well enough characterized, the volumetric strain induced by surface energy effects can therefore precisely be calculated theoretically. This calculation will be possible however only if the surface stress $\tilde{\sigma}^{s}$ which prevails at the interface between the solid matrix and the pore fluid is known. How to estimate a change of surface stress induced by a modification of the pore fluid is the topic of the next section.

\section{Surface stress and adsorption}

In order to compute the strain of the porous medium the surface stress $\tilde{\sigma}^{s}$ at the interface between solid matrix and pore fluid needs to be known. This surface stress depends on the nature and on the state of the pore fluid: Modifying the chemical potential of the pore fluid will modify the surface stress as a consequence of the adsorption of fluid molecules at the interface.

An interface is characterized by its surface energy $\gamma$, more commonly named surface tension. Surface energy (or surface tension) must not be confused with surface stress: As defined by Kramer and Weissmüller (2007), "by surface tension [...] one understands the work per area of forming new surface reversibly at constant structure, for instance by adding atoms to the surface or by cleavage." If the interface is made of solid on one side and of fluid on the other side, its surface energy $\gamma$ can be decomposed as follows:

$$
\gamma=\gamma_{\mathrm{S}}+\gamma_{\mathrm{F}}+2 W_{\mathrm{SF}}
$$

where $\gamma_{\mathrm{S}}$ is the surface energy of the solid, $\gamma_{\mathrm{F}}$ is the surface energy of the fluid, and $2 W_{\mathrm{SF}}$ is the interaction energy required in order to bring a unit area of solid in contact with a unit area of fluid.

When bringing together fluid and solid surface, adsorption -an accumulation of molecules of fluid at the interface- will occur (see figure 4). How the surface energy $\gamma$ of the interface is modified when the chemical potential $\mu$ of the fluid is modified at constant temperature $T$ is governed by the celebrated Gibbs' adsorption isotherm (Gibbs, 1928): 


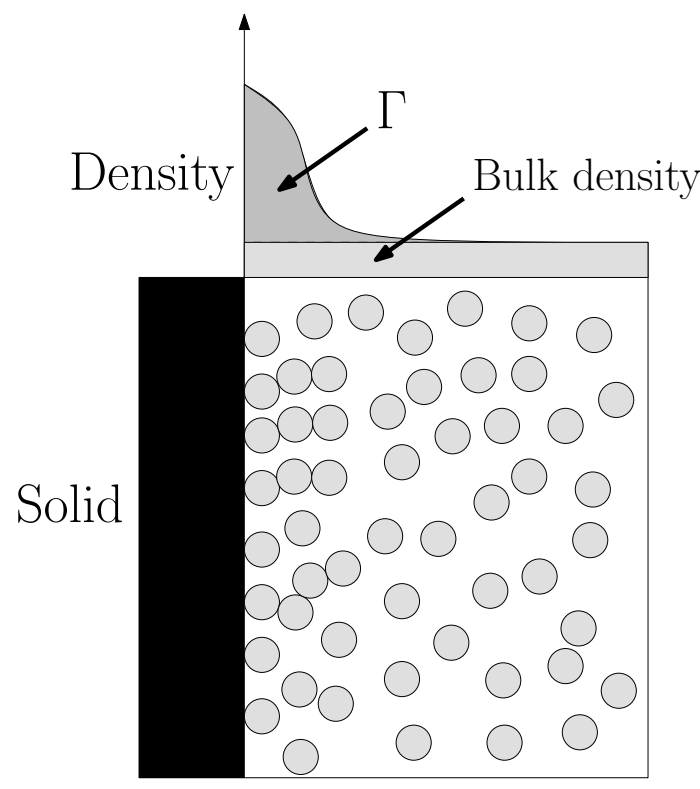

Figure 4: Adsorption of fluid molecules at an interface between solid and pore space.

$$
\mathrm{d} \gamma=-\Gamma(\mu, T) \mathrm{d} \mu
$$

where $\Gamma$ is the number of moles of fluid molecules adsorbed, in excess of the bulk density, per unit area of the interface.

Equation (43) enables to calculate a change of surface energy from a reference state, as long as the function $\Gamma(\mu, T)$, the adsorption isotherm, is known for the pore fluid considered. But what is needed in order to compute strains is the surface stress $\tilde{\sigma}^{s}$.

In this section we focus first on the link between surface energy $\gamma$ and surface stress $\tilde{\sigma}^{s}$ for a solid-vacuum interface or for a fluid-vacuum interface. Then, we will consider a solid-fluid or a fluid-fluid interface and assess how adsorption modifies the surface stress $\tilde{\sigma}^{s}$.

\subsection{Link between surface energy and surface stress in vacuum}

In this section we consider an interface between solid or fluid and vacuum. Therefore no adsorption occurs. By definition the free energy $F^{\text {surf }}$ of an 
interface of area $A$ can be increased by providing work against the surface stress $\tilde{\sigma}^{s}$ :

$$
\mathrm{d} F^{\text {surf }}=\tilde{\sigma}^{s} \mathrm{~d} A
$$

The surface energy $\gamma$ of an interface is usually measured with respect to the deformed state, i.e., in Eulerian coordinates. The very definition of this (eulerian) surface energy $\gamma$ yields the following relation:

$$
F^{\text {surf }}=\gamma A
$$

For a liquid surface at a given chemical potential no deformation of the surface can occur at fixed number of atoms: The area of a liquid surface can only be increased by bringing new liquid molecules to the surface. The surface energy remains constant, therefore $\mathrm{d} F^{\text {surf }}=\gamma \mathrm{d} A$, which after comparison with equation (44) yields:

$$
\tilde{\sigma}^{s}=\gamma
$$

from what we conclude that surface energy and surface stress are the same for a liquid surface.

A solid surface however can be strained while keeping a constant number of atoms at the surface. In order to characterize the deformation of the surface we introduce the true strain $\varepsilon_{T}$ :

$$
\varepsilon_{T}=\ln \frac{A}{A_{0}}
$$

where $A_{0}$ is the area of the undeformed surface. A differentiation of equation (45) yields:

$$
\mathrm{d} F^{\text {surf }}=\gamma \mathrm{d} A+A \mathrm{~d} \gamma=\gamma \mathrm{d} A+A \frac{\mathrm{d} \gamma}{\mathrm{d} \varepsilon_{T}} \mathrm{~d} \varepsilon_{T}=\left(\gamma+\frac{\mathrm{d} \gamma}{\mathrm{d} \varepsilon_{T}}\right) \mathrm{d} A
$$

from what follows after comparison with equation (44):

$$
\tilde{\sigma}^{s}=\gamma+\frac{\mathrm{d} \gamma}{\mathrm{d} \varepsilon_{T}}
$$

known as Shuttleworth's equation (Shuttleworth, 1950). For a solid surface, surface energy and surface stress are therefore different. 
Instead of using Eulerian coordinates however, one can work with Lagrangian coordinates and define a Lagrangian surface energy $\gamma^{L}$ with respect to the undeformed surface:

$$
F^{\text {surf }}=\gamma^{L} A_{0}
$$

A comparison of the above equation with equation (45) shows that $\gamma=$ $\gamma^{L} A_{0} / A$, which yields after replacement in equation (49):

$$
\pi^{L}=\frac{\mathrm{d} \gamma^{L}}{\mathrm{~d} \varepsilon_{T}}, \text { where } \pi^{L}=\frac{A}{A_{0}} \tilde{\sigma}^{s}
$$

This equation, already derived by Cahn (1978), is the analogous of Shuttleworth's equation (49) in Lagrangian coordinates. $\tilde{\sigma}^{s}$ and $\pi^{L}$ are for an interface the analogues of a Cauchy stress and of a Piola-Kirchhoff stress, respectively.

The Lagrangian expression (51) shows that surface stress and surface energy (or surface tension) have distinct physical meanings. Although their values are equal for a liquid surface, both experiment and theory show that their value can significantly differ from each other for a solid surface (Andrieu and Müller, 2005).

\subsection{Modification of surface stress by adsorption}

Instead of considering an interface with vacuum, we now consider a fluidfluid or a fluid-solid interface. This section is devoted to determining how adsorption modifies the surface stress.

By definition of the surface energy $\gamma$ the free energy $F^{\text {surf }}$ of an interface of area $A$ is given by:

$$
F^{\text {surf }}=\gamma A+\mu N^{\text {surf }}
$$

where $N^{\text {surf }}$ is the number of moles of adsorbed molecules of fluid in excess of the bulk density and where $\mu$ is the molar chemical potential of the adsorbed molecules. Work can be provided to the interface either by increasing its area or by adding more adsorbed molecules. For a fluid-fluid interface the area of the interface can only be increased by adding new molecules so that the energy balance for the interface is:

$$
\mathrm{d} F^{\text {surf }}=\gamma \mathrm{d} A+\mu \mathrm{d} N^{\text {surf }}
$$


A combination of the two equations above yields Gibbs' adsorption isotherm (43).

In contrast, if we consider a solid-fluid interface for which the solid surface can not be increased by adding new molecules, the energy balance for the interface becomes:

$$
\mathrm{d} F^{\text {surf }}=\tilde{\sigma}^{s} \mathrm{~d} A+\mu \mathrm{d} N^{\text {surf }}
$$

A combination of the above equation with equation (52) yields:

$$
\begin{aligned}
\tilde{\sigma}^{s} \mathrm{~d} A & =\gamma \mathrm{d} A+A \mathrm{~d} \gamma+N^{\text {surf }} \mathrm{d} \mu \\
& \Downarrow \\
\left(\tilde{\sigma}^{s}-\gamma-\left.\frac{\partial \gamma}{\partial \varepsilon_{T}}\right|_{\mu}\right) \mathrm{d} \varepsilon_{T} & =\left(\Gamma+\left.\frac{\partial \gamma}{\partial \mu}\right|_{\varepsilon_{T}}\right) \mathrm{d} \mu
\end{aligned}
$$

where $\Gamma=N^{\text {surf }} / A$ is the Eulerian amount of adsorbed molecules in excess of the bulk density per unit area. Since $\mu$ and $\varepsilon_{T}$ are two independent variables, the two terms in-between parentheses must be equal to zero. The left-hand side term yields:

$$
\tilde{\sigma}^{s}=\gamma+\left.\frac{\partial \gamma}{\partial \varepsilon_{T}}\right|_{\mu}
$$

which is a direct extension of Shuttleworth's equation (49) to solid-fluid interfaces. In contrast, the right-hand side term governs how surface energy evolves with adsorption at fixed deformation:

$$
\left.\frac{\partial \gamma}{\partial \mu}\right|_{\varepsilon_{T}}=-\Gamma
$$

and is the analogue of Gibbs' adsorption isotherm (43).

From equation (42) the surface energy can be expressed as:

$$
\gamma=\gamma_{S}+\gamma_{F}+2 W_{S F}
$$

where $\gamma_{S}=\gamma_{S}\left(\varepsilon_{T}\right)$ is the surface energy of the solid, where $\gamma_{F}=\gamma_{F}(\mu)$ is the surface energy of the fluid, and where $2 W_{S F}=2 W_{S F}\left(\varepsilon_{T}, \mu\right)$ is the work needed to bring a unit area of solid interface into contact with a unit area of 
fluid interface. Assuming that the adsorbed density $\Gamma$ does not depend on the deformation of the surface, equation (58) can be integrated as:

$$
\gamma_{F}+2 W_{S F}=-\int_{\mu=-\infty}^{\mu} \Gamma(\bar{\mu}) \mathrm{d} \bar{\mu}
$$

from what follows $W_{S F}\left(\varepsilon_{T}, \mu\right)=W_{S F}(\mu)$. Combining equations (57), (59) and (60) yields:

$$
\tilde{\sigma}^{s}=\left(\gamma_{S}+\left.\frac{\partial \gamma_{S}}{\partial \varepsilon_{T}}\right|_{\mu}\right)+\left(\gamma_{F}+2 W_{S F}\right)=\left(\gamma_{S}+\left.\frac{\partial \gamma_{S}}{\partial \varepsilon_{T}}\right|_{\mu}\right)-\int_{\mu=-\infty}^{\mu} \Gamma(\bar{\mu}) \mathrm{d} \bar{\mu}
$$

in which equation the left term is recognized as the surface stress $\tilde{\sigma}_{a}^{s}=$ $\tilde{\sigma}^{s}(N \rightarrow 0)$ that prevails on the solid side when no fluid molecule is adsorbed on the interface:

$$
\tilde{\sigma}^{s}=\tilde{\sigma}_{a}^{s}-\int_{\mu=-\infty}^{\mu} \Gamma(\bar{\mu}) \mathrm{d} \bar{\mu}
$$

In the above equation the first term takes into account the surface stress that exists on the solid side in the absence of any adsorption process while the second term captures both the surface stress that exists on the fluid side and the effect of adsorption. From a reference state in which no adsorption occurs, the change $\Delta \tilde{\sigma}^{s}$ of surface stress due to adsorption is thus given by:

$$
\Delta \tilde{\sigma}^{s}=-\int_{\bar{\mu}=-\infty}^{\mu} \Gamma(\bar{\mu}) \mathrm{d} \bar{\mu}
$$

\section{Application to swelling of coal}

We now come back to the problem of the volumetric deformation of coal consecutive to an injection of methane or carbon dioxide. Coal is a fossil fuel. It is a combustible, sedimentary, organic rock, which is composed mainly of carbon, hydrogen, and oxygen. Coal samples are classified with respect to their degree of maturity (or rank), ranging from peat (low rank) to anthracite (high rank). The more altered coal has been over the years, the more mature it is. Low rank coal has a low carbon and energy content whereas high rank coal has a high one. Coal is known to exhibit an extremely complex pore network (Bae and Bhatia, 2006; White et al., 2005), made of both 
nanopores (radius smaller than $10 \AA$ ) and mesopores (radius greater than 10 $\AA$ ), schematically displayed in figure 5 .

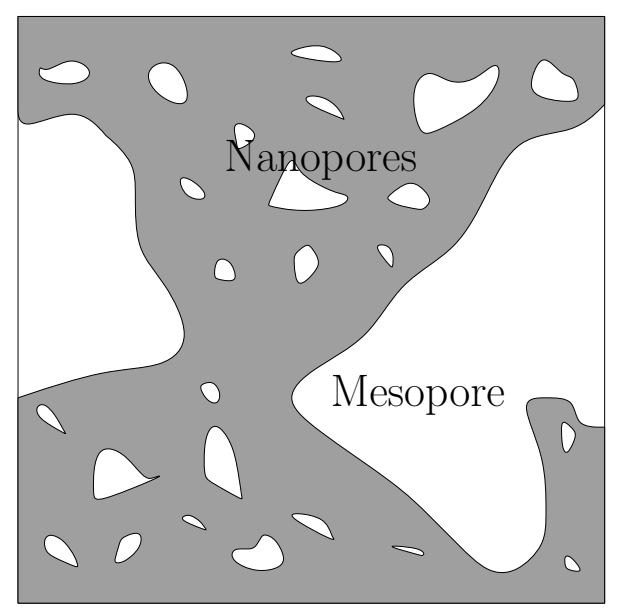

Figure 5: Schematic representation of the hierarchical pore size distribution in coal.

If the adsorbed amount of fluid is known the work performed in section 2 enables to calculate the induced surface stress. The work performed in section 1 then enables to calculate the corresponding volumetric strain at this given surface stress. For an unjacketed experiment $(\sigma=-p)$ such as the one displayed in figure $2 \mathrm{~b}$ the volumetric strain $\epsilon$ is given by equation (21):

$$
\epsilon=\epsilon_{a}-\frac{1-b}{K} p=-\alpha_{\epsilon} \tilde{\sigma}^{s}-\frac{1-b}{K} p
$$

In such an unjacketed experiment strains are measured from the reference state in which $p=0$. We note that $b=1-K / k_{s}$, where $k_{s}$ is the bulk modulus of the solid matrix (Coussy, 2004). For infinitesimal transformations we can neglect the effect of strain on the surface stress, and equation (63) enables to express the change $\Delta \epsilon$ in volumetric strain measured from the reference state as:

$$
\Delta \epsilon=-\frac{p}{k_{s}}+\alpha_{\epsilon} \int_{\bar{\mu}=-\infty}^{\mu_{F}(p)} \Gamma_{F}(\bar{\mu}) \mathrm{d} \bar{\mu}
$$

where $F$ stands for $\mathrm{CO}_{2}$ or $\mathrm{CH}_{4}$ and where $\Gamma_{F}$ is the adsorption isotherm of the pore fluid considered. Since methane has a critical temperature $T_{\mathrm{CH}_{4}}^{c}=$ 
190.6 $\mathrm{K}=-82.6^{\circ} \mathrm{C}$ and a critical pressure $P_{\mathrm{CH}_{4}}^{c}=4.60 \mathrm{MPa}$, while carbon dioxide has a critical temperature $T_{\mathrm{CO}_{2}}^{c}=304.1 \mathrm{~K}=31.0^{\circ} \mathrm{C}$ and a critical pressure $P_{\mathrm{CO}_{2}}^{c}=7.38 \mathrm{MPa}$ (Cengel and Boles, 2008), both methane and carbon dioxide may be in supercritical conditions in the field.

The computation of the strain induced by an injection of pore fluid therefore requires the determination of the adsorption isotherm (i.e., the amount of fluid adsorbed at a given chemical potential), possibly in supercritical conditions. In this section we focus on how to estimate adsorption isotherms and whether adsorption isotherms enable to correctly predict the swelling observed experimentally for both carbon dioxide and methane. The estimation of adsorption isotherm is performed with the Langmuir adsorption model and with molecular simulations.

\subsection{Use of Langmuir isotherm}

In this section we focus on the Langmuir adsorption model and on whether this model enables to correctly predict the swelling behavior observed experimentally. The Langmuir adsorption model (Langmuir, 1916) is the simplest model for adsorption. In this model a surface of finite extent contains a finite number of adsorption sites, all sites being equivalent to each other. Moreover, each adsorption site can only accommodate one molecule. The classical Langmuir adsorption isotherm links the fraction $\theta$ of occupied sites with the pressure $p$ of the pore fluid:

$$
\theta=\frac{p}{p+c_{\mathrm{F}}(T)}
$$

where $c_{\mathrm{F}}(T)$ depends on the temperature and on the nature of the adsorbed fluid ( $\mathrm{F}$ stands for $\mathrm{CO}_{2}$ or $\mathrm{CH}_{4}$ ). The adsorption isotherm (66) holds for ideal gases. In an underground coal bed however both methane and carbon dioxide may be in supercritical conditions and the Langmuir adsorption model must therefore be extended to supercritical conditions. For the general case of equivalent sites of adsorption, the molar chemical potential $\mu^{a}$ of the adsorbed molecules of fluid is given by (Fowler, 1935):

$$
\mu^{a}=R T \ln \left(\frac{\theta}{1-\theta}\right)+f_{\mathrm{F}}(T)
$$

where $f_{F}(T)$ is a function of temperature and of the nature of the adsorbed fluid. At equilibrium the chemical potential of the adsorbed molecules must 
equal that of the pore fluid, which enables to express the fraction of occupied sites as a function of the chemical potential $\mu_{\mathrm{F}}$ of the pore fluid:

$$
\theta=\frac{1}{1+f_{\mathrm{F}}(T) e^{-\mu_{\mathrm{F}} /(R T)}}
$$

where $\mathrm{F}$ stands again for $\mathrm{CO}_{2}$ or $\mathrm{CH}_{4}$. For the specific case of an ideal gas, for which $\mu_{\mathrm{F}}=\mu_{\mathrm{F}}^{0}+R T \ln \left(p / p^{0}\right)$, the above formula reduces as expected to the usual Langmuir isotherm (66).

Equation (68) then enables to calculate the number $\Gamma$ of molecules per unit area adsorbed in excess of the bulk density:

$$
\Gamma=\Gamma_{\mathrm{F}}^{\max } \theta_{\mathrm{F}}=\frac{\Gamma_{\mathrm{F}}^{\max }}{1+f_{\mathrm{F}} e^{-\mu_{\mathrm{F}} /(R T)}}
$$

where $\Gamma_{\mathrm{F}}^{\max }$ and $f_{\mathrm{F}}$ are two parameters which determine the shape of the adsorption isotherm for each pore fluid. A combination of equations (65) and (69) eventually enables to express the volumetric strain due to an injection of fluid:

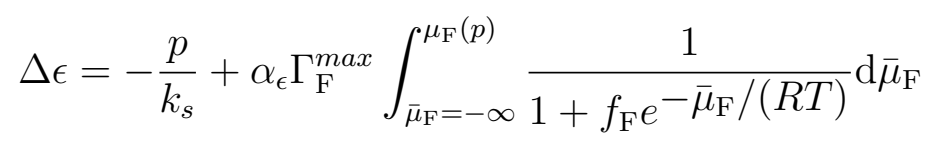

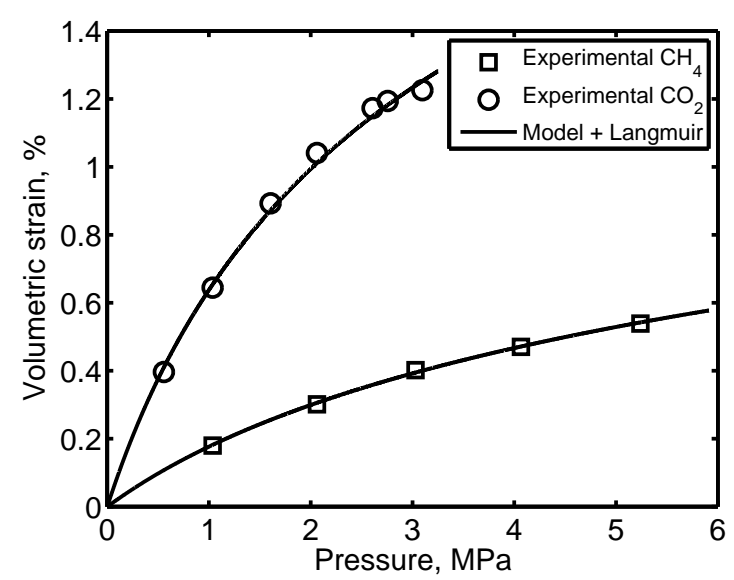

Figure 6: Volumetric strain predicted with the Langmuir adsorption isotherms (with experimental data from Levine (1996)). 
After a tremendous work having consisted in fitting equations of state to very large experimental datasets, Span and Wagner (2003a, 2003b) made the equations of state for methane and carbon dioxide available even in supercritical conditions. In figure 6 we display the best fit obtained with equation (70) and the equations of state of Span and Wagner. The model fits very well the experimental data. The best fits were for $\alpha_{\epsilon} \Gamma_{\mathrm{CH}_{4}}^{\max }=1.76 \times 10^{-6}$ mol. $\mathrm{N}^{-1} . \mathrm{m}^{-1}, f_{\mathrm{CH}_{4}}=20.3, \alpha_{\epsilon} \Gamma_{\mathrm{CO}_{2}}^{\max }=3.88 \times 10^{-6} \mathrm{~mol} . \mathrm{N}^{-1} \cdot \mathrm{m}^{-1}, f_{\mathrm{CO}_{2}}=10.4$, and $k_{s}=94.4 \mathrm{GPa}$.

The very high value for $k_{s}$ shows that the compressibility of the coal solid matrix itself has here little effect on the overall deformation. Interestingly, the bulk modulus $K$ of coal as a porous medium is only of 1 to $3 \mathrm{GPa}$ (Wang et al., 2009), i.e., much lower than that of the solid matrix and thus of a coal in which there would be neither nanopores nor mesopores: The overall porosity of coal, which goes down to the Angstrom scale, has a tremendous effect on its mechanical properties.

The fitted parameters yielded $\Gamma_{\mathrm{CO}_{2}}^{\max } / \Gamma_{\mathrm{CH}_{4}}^{\max } \approx 2$. The fact that a given mass of coal can adsorb much more carbon dioxide than methane has already been observed experimentally (Stanton et al., 2001).

The molecular volumes of methane and carbon dioxide are almost identical, approximately $30 \AA^{3}$ (Webster et al., 1998). If a single layer of molecules could get adsorbed, the maximum density of adsorbed molecules (full monolayer) would be $\Gamma=1.7 \times 10^{-5} \mathrm{~mol} . \mathrm{m}^{-2}$ and therefore we would have $\alpha_{\epsilon} \approx 0.1-0.2 \mathrm{~N}^{-1}$.m. If we assume that the coal pore system is made of spherical mono-sized pores, equation (28) would then yield $K R \approx 10 \mathrm{~N}^{-\mathrm{m}^{-1}}$. The bulk modulus of coal being on the order of 1 GPa to 3 GPa (Wang et al., 2009), the model we developed would provide the right magnitude of strain if the coal pores have a radius on the order of 3 to $10 \mathrm{~nm}$, i.e., would fall in the range of mesopores.

The fact that the Langmuir adsorption model, which is the simplest model for adsorption, provides such a good fit of the experimental swelling data is intriguing. For instance, the Langmuir adsorption model assumes that a unique layer of molecules can get adsorbed, which is unlikely to happen in mesopores since the radius of a mesopore is much greater than the size of a fluid molecule. In order to gain a better insight in the physics of the adsorption process and in order to understand why the Langmuir model works so well in our case, we perform in the next section molecular simulations of adsorption of carbon dioxide and methane in coal. The adsorption isotherms obtained from the simulations (in both mesopores and nanopores) are then 
used for calculation of swelling.

\subsection{Implementation of molecular simulations}

We performed Monte Carlo molecular simulations of adsorption in the Grand Canonical statistical ensemble (Frenkel and Smit, 2001): The temperature $T$ of the system, the volume $V$ of the box of simulation, and the chemical potentials $\mu$ of the adsorbed species (carbon dioxide or methane, depending on the simulation run) were held constant. We used periodic boundary conditions and the Metropolis algorithm (Metropolis et al., 1953).

Many varieties of coal exist, and a molecular model that reflects all types of coal is unrealistic. However, a satisfactory model should possess some average characteristics (e.g., density, pore size distribution, chemical composition) that are in accordance with the characteristics of an intermediary rank coal. A good candidate for that purpose is the CS1000 model (Jain et al., 2006), which we therefore used for our simulations (see figure 7a). The CS1000 model is the molecular representation of a high density porous saccharose coke obtained by pyrolyzing pure saccharose at $1000^{\circ} \mathrm{C}$ in a nitrogen flow. CS1000 is a cube with a side length of $25 \AA$ : The CS1000 structure is therefore unable to exhibit features with a characteristic size greater than $12.5 \AA$.

Interaction potentials involving $\mathrm{CH}_{4}, \mathrm{CO}_{2}$, and coal were defined with models available in the literature. The chosen models were selected because of their representativeness of the underlying physical interactions between molecules. A sumary of these interaction potentials is presented in table 1. Molecules were interacting according to the minimum image convention (Frenkel and Smit, 2001).

Methane-methane interactions were modeled with a Lennard-Jones potential:

$$
U_{\mathrm{CH}_{4}-\mathrm{CH}_{4}}(r)=4 \varepsilon_{\mathrm{CH}_{4}}\left(\left(\frac{\sigma_{\mathrm{CH}_{4}}}{r}\right)^{12}-\left(\frac{\sigma_{\mathrm{CH}_{4}}}{r}\right)^{6}\right)
$$

where $\sigma_{\mathrm{CH}_{4}}$ (Van der Waals radius) and $\varepsilon_{\mathrm{CH}_{4}}$ are the Lennard-Jones parameters and $r$ is the distance between the centers of the carbon atoms. The values for the parameters are from Kurniawan et al. (2006), who simulated supercritical methane, and are given in table 1 . The potential was truncated at a distance of $23 \AA$, which is reasonable regarding the value of $\sigma_{\mathrm{CH}_{4}}$. 
a)

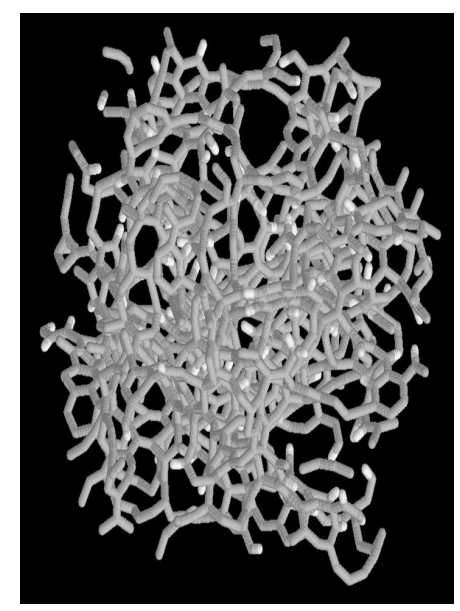

b)

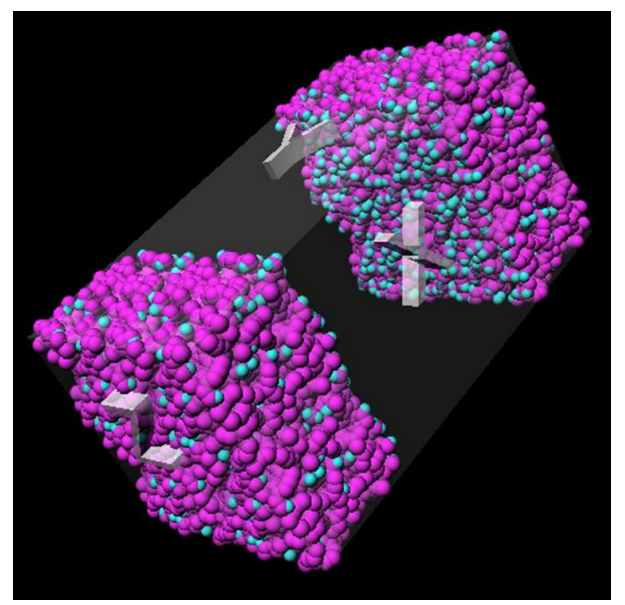

Figure 7: Box of simulation used for (a) nanopores and (b) mesopores. The box of simulation for nanopores is an unaltered CS1000 sample: Carbon atoms are gray, hydrogen atoms are white. The box of simulation for mesopores is made of eight CS1000 samples: Carbon atoms are pink, hydrogen atoms are green.

In order to capture $\mathrm{CO}_{2}-\mathrm{CO}_{2}$ interactions, the $\mathrm{CO}_{2}$ molecule was modeled with the EPM model (Harris and Yung, 1995), in which the molecule is represented by three Lennard-Jones centers (one for each atom) and three point charges at the same centers. The point charges represent the experimentally known quadrupole moment of $\mathrm{CO}_{2}$. The energy interaction is:

$$
U_{\mathrm{CO}_{2}-\mathrm{CO}_{2}}=\sum_{a=1}^{3} \sum_{b=1}^{3}\left(4 \varepsilon_{a b}\left[\left(\frac{\sigma_{a b}}{r_{a b}}\right)^{12}-\left(\frac{\sigma_{a b}}{r_{a b}}\right)^{6}\right]+\frac{1}{4 \pi \epsilon_{0}} \frac{q_{a} q_{b}}{r_{a b}}\right)
$$

where $\left(r_{a b}\right)_{a, b=\{1,2,3\}}$ is the distance between the Lennard-Jones centers of the molecules; $\sigma_{a b}$ and $\varepsilon_{a b}$ are the Lennard-Jones parameters for the interaction between atom $a$ and atom $b ; q_{a}$ and $q_{b}$ are the charges at the Lennard-Jones centers. The value of the parameters are from Harris and Yung (1995) and are given in table 1. The EPM model includes electrostatic interactions with a larger range of interaction than those of the Lennard-Jones potential. However, even if electrostatic interactions evolve as $r^{-1}$, at large distances the two 
negative charges of the oxygen atoms cancel the positive charge of the carbon. For large $r$ the first order approximation of electrostatic interaction behaves like a quadrupole-quadrupole interaction (Allen and Tildesley, 1989). The long range contribution of the electrostatic interactions decreases proportionally to $r^{-5}$ whereas the Lennard-Jones potential decreases proportionally to $r^{-6}$. Here, however, the range of quadrupole-quadrupole interactions is limited and can be neglected for distances larger than $23 \AA$; so we truncated the interaction potential at the same radius as for the methane Lennard-Jones model.

For the interaction of methane with coal we used a Lennard-Jones model:

$$
U_{\mathrm{CH}_{4}-i}=4 \varepsilon_{\mathrm{CH}_{4}-i}\left(\left(\frac{\sigma_{\mathrm{CH}_{4}-i}}{r}\right)^{12}-\left(\frac{\sigma_{\mathrm{CH}_{4}-i}}{r}\right)^{6}\right)
$$

where $i=\mathrm{C}$ or $\mathrm{H}$ is an atom of the CS1000 model. The Lennard-Jones parameters $\sigma_{\mathrm{CH}_{4}-i}$ and $\varepsilon_{\mathrm{CH}_{4}-i}$ were obtained following the Lorentz-Berthelot rules : $\sigma_{i j}=\left(\sigma_{i}+\sigma_{j}\right) / 2$ and $\varepsilon_{i j}=\sqrt{\varepsilon_{i} \varepsilon_{j}}$ where $i$ stands for $\mathrm{CH}_{4}$, and $j$ for $\mathrm{C}$ or H. $\sigma_{\mathrm{CH}_{4}}$ and $\varepsilon_{\mathrm{CH}_{4}}$ were those of the model of methane. The parameters for the carbon and hydrogen atoms of CS1000 are from Jain et al. (2006), who simulated argon adsorption in CS1000, and are given in table 1. Akin to methane, argon is a spherical non-polar molecule which can be represented with a Lennard-Jones model.

Regarding the $\mathrm{CO}_{2}$-coal interactions we had to account for the electrostatic interaction in addition to the Lennard-Jones interaction. The electrons in CS1000 have a distribution probability determined by their quantum wave functions. Because of the difference in electronegativity between atoms in CS1000, these wave functions are not centered on the nuclei but are displaced toward the most electronegative atom. A molecule of gas approaching the solid feels an electrical field which can be represented by point charges at the center of each atom of the CS1000 model. We used the "partial equalization of orbital electronegativity' method in order to estimate those point charges (Gasteiger and Marsili, 1980).

We want to estimate the adsorption behavior in both nanopores and mesopores. The nanopores are accounted for in the CS1000 model; so simulations on a CS1000 sample directly provided an estimation of the adsorption behavior in nanopores. In order to estimate the adsorption behavior in mesopores, for which curvature is negligible, we simulated adsorption on a flat surface. The flat surface was created by juxtaposing several CS1000 samples (see figure $7 \mathrm{~b}$ ). In this process most of the carbon atoms at the surface lost covalent 


\begin{tabular}{|c|c|}
\hline $\begin{array}{c}\text { Interacting } \\
\text { elements }\end{array}$ & $\begin{array}{l}\text { Interaction } \\
\text { potential }\end{array}$ \\
\hline \multirow[t]{2}{*}{$\mathrm{CH}_{4}-\mathrm{CH}_{4}$} & $U_{\mathrm{CH}_{4}-\mathrm{CH}_{4}}(r)=4 \varepsilon_{\mathrm{CH}_{4}}\left(\left(\frac{\sigma_{\mathrm{CH}_{4}}}{r}\right)^{12}-\left(\frac{\sigma_{\mathrm{CH}_{4}}}{r}\right)^{6}\right)$ \\
\hline & $\sigma_{\mathrm{CH}_{4}}=3.751 \AA ; \varepsilon_{\mathrm{CH}_{4}}=148 \times k_{B}$ \\
\hline \multirow[b]{2}{*}{$\mathrm{CO}_{2}-\mathrm{CO}_{2}$} & $\begin{array}{c}U_{\mathrm{CO}_{2}-\mathrm{CO}_{2}}=\sum_{a=1}^{3} \sum_{b=1}^{3}\left(4 \varepsilon_{a b}\left[\left(\frac{\sigma_{a b}}{r_{a b}}\right)^{12}-\left(\frac{\sigma_{a b}}{r_{a b}}\right)^{6}\right]+\frac{1}{4 \pi \epsilon_{0}} \frac{q_{a} q_{b}}{r_{a b}}\right) \\
\text { with } a, b=\mathrm{C} \text { or } \mathrm{O} \text { of } \mathrm{CO}_{2}\end{array}$ \\
\hline & $\begin{array}{c}\epsilon_{\mathrm{C}-\mathrm{C}}=28.129 \times k_{B} ; \sigma_{\mathrm{C}-\mathrm{C}}=2.757 \AA ; \epsilon_{\mathrm{O}-\mathrm{O}}=80.507 \times k_{B} \\
\sigma_{\mathrm{O}-\mathrm{O}}=3.033 \AA ; \epsilon_{\mathrm{C}-\mathrm{O}}=47.588 \times k_{B} ; \sigma_{\mathrm{C}-\mathrm{O}}=2.892 \AA \\
\text { Distance } \mathrm{C}-\mathrm{O}=1.149 \AA ; q_{\mathrm{C}}=-2 q_{\mathrm{O}}=0.6512 e\end{array}$ \\
\hline \multirow[b]{2}{*}{$\mathrm{CH}_{4}-\mathrm{Coal}$} & $\begin{array}{c}U_{\mathrm{CH}_{4}-i}=4 \varepsilon_{\mathrm{CH}_{4}-i}\left(\left(\frac{\sigma_{\mathrm{CH}_{4}-i}}{r}\right)^{12}-\left(\frac{\sigma_{\mathrm{CH}_{4}-i}}{r}\right)^{6}\right) \\
\text { with } i=\mathrm{C} \text { or H of CS1000 }\end{array}$ \\
\hline & $\begin{array}{c}\sigma_{\mathrm{CH}_{4}-i}=\left(\sigma_{\mathrm{CH}_{4}}+\sigma_{i}\right) / 2 \text { and } \varepsilon_{\mathrm{CH}_{4}-i}=\sqrt{\varepsilon_{\mathrm{CH}_{4}} \varepsilon_{i}} \\
\sigma_{\mathrm{CH}_{4}}=3.751 \AA ; \varepsilon_{\mathrm{CH}_{4}}=148 \times k_{B} ; \sigma_{\mathrm{H}}=2.42 \AA \\
\varepsilon_{\mathrm{H}}=15.08 \times k_{B} ; \sigma_{\mathrm{C}}=3.36 \AA ; \varepsilon_{\mathrm{C}}=28 \times k_{B}\end{array}$ \\
\hline \multirow[b]{2}{*}{$\mathrm{CO}_{2}-\mathrm{Coal}$} & $\begin{array}{c}U_{\mathrm{CO}_{2}-i}=\sum_{a=1}^{3}\left(4 \varepsilon_{a-i}\left(\left(\frac{\sigma_{a-i}}{r_{a i}}\right)^{12}-\left(\frac{\sigma_{a-i}}{r_{a i}}\right)^{6}\right)+\frac{1}{4 \pi \epsilon_{0}} \frac{q_{a} q_{i}}{r_{a i}}\right) \\
\text { with } a=\mathrm{C} \text { or } \mathrm{O} \text { of } \mathrm{CO}_{2} \text { and } i=\mathrm{C} \text { or H of } \mathrm{CS} 1000\end{array}$ \\
\hline & $\begin{array}{c}\sigma_{a-i}=\left(\sigma_{a}+\sigma_{i}\right) / 2 \text { and } \varepsilon_{a-i}=\sqrt{\varepsilon_{a} \varepsilon_{i}} \\
\sigma \text { and } \varepsilon \text { parameters for } \mathrm{CO}_{2}: \text { those of } \mathrm{CO}_{2}-\mathrm{CO}_{2} \\
\sigma \text { and } \varepsilon \text { parameters for } \mathrm{C} \text { and } \mathrm{H} \text { of } \mathrm{CS} 1000: \text { those of } \mathrm{CH}_{4}-\text { Coal } \\
\text { Charges } q_{i} \text { in CS1000 : } \\
\text { obtained with the PEOE method (Gasteiger and Marsili, 1980) }\end{array}$ \\
\hline
\end{tabular}

Table 1: Summary of interaction potentials. $k_{B}$ is the Boltzmann constant.

bonds. In order to ensure the electroneutrality of the sample, similarly to what was done by Pellenq and Levitz (2001), for each covalent bond lost we positioned supplementary hydrogen atoms at a distance of $1.09 \AA$ (optimum bond length for the C-H bond (Jain et al., 2006)) from the carbon atom in the direction of the former covalent bond. The atoms of the solid matrix remained fixed during the simulations. 


\subsection{Calculations and results of molecular simulations}

The adsorption isotherms were calculated at $310 \mathrm{~K}$ for pressures up to 20 $\mathrm{MPa}$ for both methane and carbon dioxide. An example of state is displayed in figure 8. Both nanopores and mesopores (flat interface) were considered.

The calculation of an adsorption isotherm requires to express amounts of fluid molecules adsorbed in excess of the bulk density by unit area of the interface. This calculation was straightforward for the adsorption in mesopores. For the adsorption in nanopores we had to determine the volume of those few-Angstrom-large pores as well as an equivalent area of the interface. The volume of nanopores was estimated with the maximum amount of $\mathrm{CO}_{2}$ molecules at $20 \mathrm{MPa}$ (this amount was nearly constant for larger pressures). Those molecules were assumed to be at a liquid-like density $\left(\approx 1.45 \times 10^{-2}\right.$ molecule. $\left.\AA^{-3}\right)$ which yielded a volume of nanopores in the CS1000 of $1.13 \times 10^{4} \AA^{3}$. The same calculation with the maximum amount of methane yielded a volume of $1.09 \times 10^{4} \AA^{3}$. Each of those molecules of carbon dioxide was then considered to cover a surface of $16.9 \AA^{2}$ (liquid density) which corresponded to a total surface of nanopores of $27.4 \mathrm{~nm}^{2}$ in the CS1000. The same calculation with the methane molecules yielded a total surface of nanopores of $25.3 \mathrm{~nm}^{2}$.

The results for both nanopores and mesopores are displayed in figure 9 . Note that the isotherms are excess amounts of molecules, i.e., molecules in excess of the bulk density. In both nanopores and mesopores the adsorbed amount of methane increases with pressure and saturates at high pressures. For carbon dioxide however the adsorption isotherms are not monotonic functions: Around $8 \mathrm{MPa}$ the critical pressure is reached, which results in a significant increase in bulk density and thus in a significant decrease of the amount adsorbed in excess. At subcritical pressures the size of the pores significantly modifies the adsorption behavior of carbon dioxide in coal: In a nanopore the adsorption behavior is a convex function of pressure while in a mesopore the adsorbed amount is a concave function of pressure. For any type of pore, at pressures below $8 \mathrm{MPa}$, carbon dioxide is adsorbed in greater amounts than methane.

\subsection{Discussion}

The calculated isotherms and equation (65) enable to calculate the volumetric strain induced by the pore fluid. Based on the adsorption isotherm in 
a)

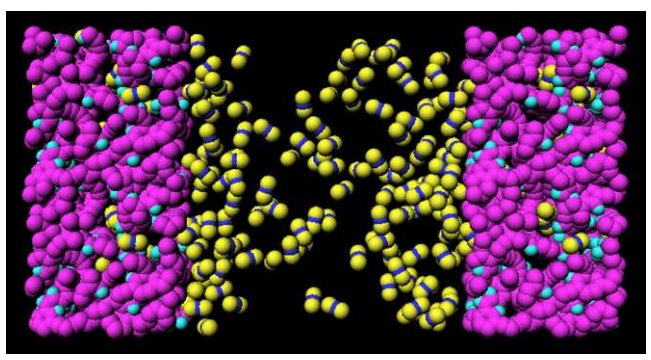

b)

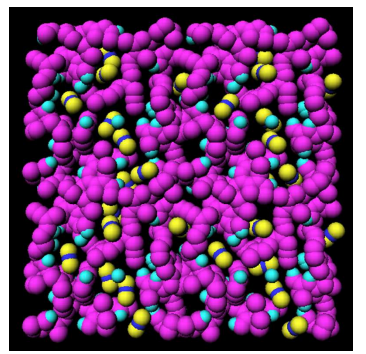

Figure 8: Examples of states obtained from molecular simulations: Adsorption of carbon dioxide (a) in a mesopore and (b) in nanopores. Oxygen atoms are yellow. Carbon atoms of the CS1000 are pink.

mesopores, the best fit for the volumetric strain is obtained for an incompressible solid matrix $\left(k_{s} \rightarrow+\infty\right)$ and $\alpha_{\epsilon}=1.67 \mathrm{~N}^{-1} . \mathrm{m}$ (figure 10a); while with the adsorption isotherm in nanopores the best fit is obtained for an incompressible matrix $\left(k_{s} \rightarrow+\infty\right)$ and $\alpha_{\epsilon}=1.71 \times 10^{-1} \mathrm{~N}^{-1}$.m (figure 10b).

The swelling predicted with adsorption isotherms in mesopores fits the experimental data very poorly: At low pressures the model predicts a swelling that does not depend on the adsorbed fluid (methane or carbon dioxide). This stems from the fact that at the lowest pressures methane and carbon dioxide get adsorbed in equivalent amounts on a flat surface (figure 9). The differential swelling of coal (why coal swells more with carbon dioxide than with methane at a given pore pressure) can therefore not be explained by adsorption in mesopores.

In contrast, the swelling predicted with adsorption isotherms in nanopores (figure 10b) is in better agreement with experimental data, since it captures that an adsorption of carbon dioxide yields a greater strain than an adsorption of methane, especially at low pressures. This comparison shows that nanopores play a primordial role in the adsorption behavior of coal. Nanopores are so small that they can only welcome a couple of fluid molecules, thus behaving as well-defined adsorption sites that get saturated above a given pressure, which can explain why the Langmuir adsorption model provides such a good fit of the experimental swelling data and why the material parameter $\alpha_{\epsilon}$ obtained with the adsorption isotherm in nanopores $\left(\alpha_{\epsilon}=1.71 \times 10^{-1} \mathrm{~N}^{-1}\right.$.m) falls in the range obtained with the Langmuir isotherm $\left(\alpha_{\epsilon} \approx 0.1-0.2 \mathrm{~N}^{-1} . \mathrm{m}\right.$, see section 3.1). 


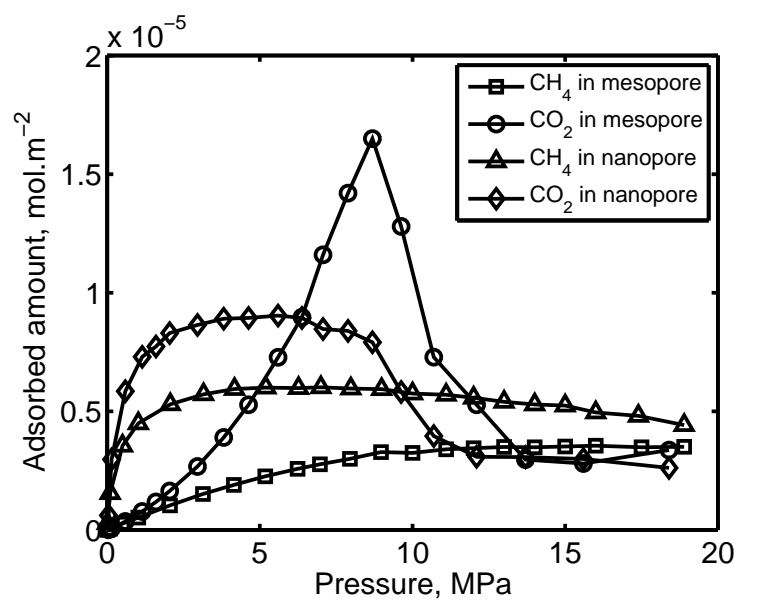

Figure 9: Excess amounts of methane and carbon dioxide adsorbed in coal in a mesopore (flat surface) and in nanopores.

Like for Gibbs' theory the relevant parameter is the amount of adsorbed molecules in excess per unit area of the interface. The use of such a thermodynamics therefore requires to determine the area of the interface. For a flat surface such a determination is straightforward. In contrast, determining the surface area of a nanopore is a much more complex question, and different methods will yield different results. But, for the smallest pores, which can only welcome one or two fluid molecules, the concept of surface may even become irrelevant. In nanopores adsorption occurs by pore filling more than by surface covering (Dubinin, 1966), which can explain why the characteristic pore size back-calculated with the Langmuir isotherm was wrong in suggesting that adsorption mainly happens in mesopores (see section 3.1).

An issue with Monte Carlo molecular simulations is that they give access to equilibrium states only. No information about kinetics or pore accessibility is obtained, which may be unfortunate for the practical case of coal. As mentioned in section 2, a given mass of coal can adsorb at least twice as much (in moles per unit mass of coal) carbon dioxide as methane. This experimental observation is tentatively explained by activated diffusion (Anderson et al., 1965, Walker and Geller, 1956): Carbon dioxide molecules would be able to penetrate within the coal matrix (by elastically deforming it locally?) and thus reach pores that remain closed for methane molecules. In the molecular simulations here performed both carbon dioxide and methane fluid molecules 
a)

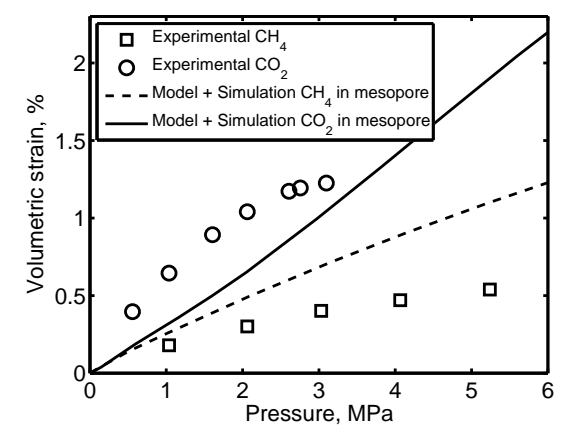

b)

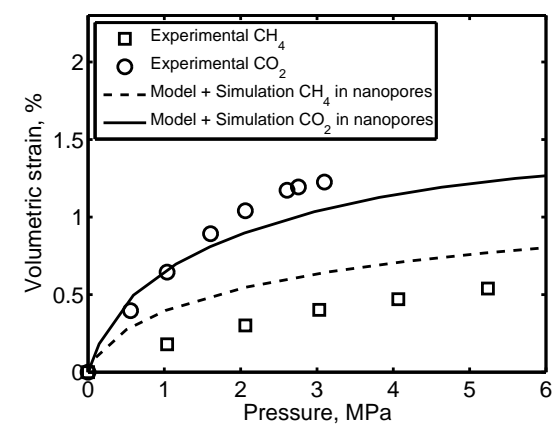

Figure 10: Volumetric strain predicted with the adsorption isotherms obtained by molecular simulation of adsorption in coal (a) in a mesopore, i.e., on a flat surface, and (b) in nanopores.

had access to all nanopores. In order to gain insight into the issue of activated diffusion, more information is required about the energy barrier a fluid molecule needs to overcome in order to access a remote nanopore. The simulations here performed provide no such information.

The coefficient $\alpha_{\epsilon}$ (or $\alpha_{\varphi}$ ) was obtained by linearization under the assumption of infinitesimal transformations. The fact that the developed framework, combined with the Langmuir adsorption model, captured very well the experimental data suggests that the linearization was valid, meaning that the pore surface is only infinitesimally deformed by adsorption effects.

\section{Conclusions}

In this work we developed a framework that enables to calculate a macroscopic strain caused by an adsorption of fluid at the pore surface of a porous medium. The calculation of the macroscopic strain requires the knowledge of the adsorption isotherm for the fluid considered. The adsorption isotherm can be either determined experimentally or directly estimated from molecular simulations thus bypassing the usual experimental difficulties. The framework developed herein therefore provides a way of calculating macroscopic strains from results obtained at the molecular scale directly. Molecular simulations also provided an interesting insight into the physics of adsorption: 
They showed that nanopores play a primordial role in the adsorption behavior of coal.

The thermodynamics of interfaces enabled to make the link between adsorption behavior and macroscopic deformations. We assumed that the thermodynamics of interfaces remained a valid tool even at the smallest scales, but this is likely not to be the case: in nanopores, which can only welcome a few molecules, do the notions of interface surface stress, or even of pressure, still hold? How nanopore filling leads to deformation may require a new thermodynamics to be developed.

How surface stress and macroscopic deformation are linked was derived by extending the realm of poromechanics to surface energy. The derivation is valid for any microstructure. The parameters needed in order to compute the adsorption strain can be measured by macroscopic testing. Their calculation is also theoretically possible as soon as the microstructure is fully characterized in terms of spatial organization and elastic properties. In order to do so, micromechanics can be of great help.

The developments performed in this work were compared with laboratory experimental data on the swelling of a coal sample injected with a pore fluid. They should ultimately be compared with field measurements of injectivity over time during injection in coal bed reservoirs. This latter comparison requires an extension of our work from the scale of the material up to the scale of the reservoir by introducing the cleat system and determining the impact of coal swelling on the cleats permeability.

\section{Acknowledgments}

Our thoughts go to Pr. Coussy, who played a key role in this research. His loss is invaluable to us, scientifically and personally.

Schlumberger is thankfully acknowledged for its financial support. Rolland Pellenq is gratefully acknowledged for his invaluable help in the implementation of the numerical simulations.

\section{References}

Allen, M.P., Tildesley, D.J., 1989. Computer Simulation of Liquids. Oxford University Press, Inc., New York, NY. 
Anderson, R.B., Bayer, J., Hofer, L.J.E., 1965. Determining surface areas from CO2 isotherms. Fuel 44, 443-452.

Andrieu, A., Müller, P., 2005. Les surfaces solides: concepts et méthodes, Paris.

Bae, J.S., Bhatia, S.K., 2006. High-pressure adsorption of methane and carbon dioxide on coal. Energy \& Fuels 20 (6), 2599-2607.

Bangham, D.H., Maggs, F.A.P., 1943. The strength and elastic constants of coals in relation to their ultra-fine structure. Conference on the ultra-fine structure of coals and cokes. British Coal Utilization Research Association, London, pp. 118-130.

Busch, A., Krooss, B.M., Gensterblum, Y., Van Bergen, F., Pagnier, H.J.M., 2003. High-pressure adsorption of methane, carbon dioxide and their mixtures on coals with a special focus on the preferential sorption behaviour. Journal of Geochemical Exploration 78-79, 671-674.

Cahn, J.W., 1978. Thermodynamics of solid and fluid surfaces. In: Johnson, W.C., Blakely, J.M. (Eds.), Segregation to interfaces. ASM, Metals Park, OH, pp. 3-23.

Cengel, Y.A., Boles, M.A., 2006. Thermodynamics: an engineering approach, New York, NY.

Coussy, O., 2004. Poromechanics. Wiley, Hoboken, NJ.

Dormieux, L., Kondo, D., Ulm, F.-J., 2006. Microporomechanics. Wiley, Hoboken, NJ.

Dubinin, M.M., 1966. Chemistry and Physics of Carbon, vol. 2. Marcel Dekker, New York, NY.

Fowler, R.H., 1935. A statistical derivation of Langmuir's adsorption isotherm. Mathematical Proceedings of the Cambridge Philosophical Society 31 (2), 260-264.

Frenkel, D., Smit, B., 2001. Understanding Molecular Simulation. Academic Press, Inc., San Diego, CA.

Gan, H., Nandi, S.P., Walker, P.L., 1972. Nature of the porosity in american coals. Fuel 51, 272-277.

Gasteiger, J., Marsili, M., 1980. Iterative partial equalization of orbital electronegativity - a rapid access to atomic charges. Tetrahedron 36 (22), 3219-3228.

Gibbs, J.W., 1928. The Collected Works of J. Willard Gibbs. Longmans, Green and Co, New York, NY.

Harpalani, S., Schraufnagel, A., 1990. Measurement of parameters impacting methane recovery from coal seams. Geotechnical and Geological 
Engineering 8 (4), 369-384.

Harris, J.G., Yung, K.H., 1995. Carbon dioxides liquid-vapor coexistence curve and critical properties as predicted by a simple molecular-model. Journal of Physical Chemistry 99 (31), 12021-12024.

Jain, S.K., Gubbins, K.E., Pellenq, R.J.M., Pikunic, J.P., 2006. Molecular modeling and adsorption properties of porous carbons. Carbon 44 (12), 2445-2451.

Jain, S.K., Pellenq, R.J.M., Pikunic, J.P., Gubbins, K.E., 2006. Molecular modeling of porous carbons using the hybrid reverse Monte Carlo method. Langmuir 22 (24), 9942-9948.

Jenkins, C.D., Boyer, C.M., 2008. Coalbed- and shale-gas reservoirs. Journal of Petroleum Technology 60 (2), 92-99.

Kowalczyk, P., Ciach, A., Neimark, A. V., 2008. Adsorption-induced deformation of microporous carbons: Pore size distribution effect. Langmuir 24, 6603-6608.

Kramer, D., Weissmüller, J., 2007. A note on surface stress and surface tension and their interrelation via Shuttleworth's equation and the Lippmann equation. Surface Science 601, 3042-3051.

Kurniawan, Y., Bhatia, S.K., Rudolph, V., 2006. Simulation of binary mixture adsorption of methane and $\mathrm{CO} 2$ at supercritical conditions in carbons. Aiche Journal 52 (3), 957-967.

Langmuir, I., 1916. The Evaporation, Condensation and Reflection of Molecules and the Mechanism of Adsorption. Physical Review 8 (2), 149.

Laplace, P.S., 1843-1846. Traité de Mécanique Céleste. Editions Jacques Gabay.

Levine, J.R., 1996. Model study of the influence of matrix shrinkage on absolute permeability of coal bed reservoirs. Geological Society, London, Special Publications 109 (1), 197-212.

Metropolis, N., Rosenbluth, A.W., Rosenbluth, A.H., Teller, A.H., Teller, E., 1953. Equations of state calculations by fast computing machines. Journal of Chemical Physics 21 (6), 1087-1092.

Palmer, I., Mansoori, J., 1998. How permeability depends on stress and pore pressure in coalbeds: a new model. SPE Reservoir Evaluation and Engineering, 539-544.

Pan, Z.J., Connell, L.D., 2007. A theoretical model for gas adsorptioninduced coal swelling. International Journal of Coal Geology 69 (4), 243-252.

Pellenq, R.J.M., Levitz, P.E., 2001. Adsorption/condensation of xenon in a disordered silica glass having a mixed (micro a mixed and meso) porosity. 
Molecular Simulation 27 (5-6), 353-370.

Reeves, S.R., 2004. The Coal-Seq project: key results from field, laboratory and modeling studies. In: Wilson, M., Rubin, E.S., Weith, D.W., Gilboy, C.F., Morris, T., Thambimuthu, K., Gale, J. (Eds.), 7th International Conference on Greenhouse Gas Control Technologies, Vancouver, BC, Canada.

Scherer, G.W., 1986. Dilatation of porous glass. Journal of the American Ceramic Society 69 (6), 473-480.

Shuttleworth, R., 1950. The surface tension of solids. Proceedings of the Physical Society. Section A 63, 444-457.

Span, R., Wagner, W., 2003. Equations of state for technical applications. II. Results for nonpolar fluids. International Journal of Thermophysics 24 (1), 41-109.

Span, R., Wagner, W., 2003. Equations of state for technical applications. III. Results for polar fluids. International Journal of Thermophysics 24 (1), 111-162.

Stanton, R.W., Burruss, R.C., Flores, R.M., Warwick, P.D., 2001. CO2 adsorption in low-rank coals: Progress toward assessing the nation-wide capacity to store CO2 in the subsurface. Eighteenth Annual International Pittsburg Coal Conference, Newcastle, NSW, Australia, pp. 1-2.

Van Bergen, F., Winthaegen, P., Pagnier, H., Krzystolik, P., Jura, B., Skiba, J., Van Wageningen, N., 2009. Assessment of CO2 storage performance of the enhanced coalbed methane pilot site in Kaniow. Energy Procedia 1 (1), 3407-3414.

Walker, P.L., Geller, I., 1956. Change in surface area of anthracite on heat treatment. Nature 178, 1001-1001.

Wang, G.X., Massarotto, P., Rudolph, V., 2009. An improved permeability model of coal for coalbed methane recovery and $\mathrm{CO} 2$ geosequestration. International Journal of Coal Geology 77 (1-2), 127-136.

Webster, C.E., Drago, R.S., Zerner, M.C., 1998. Molecular dimensions for adsorptives. Journal of the American Chemical Society 120 (22), 5509-5516.

White, C.M., Smith, D.H., Jones, K.L., Goodman, A.L., Jikich, S.A., LaCount, R.B., DuBose, S.B., Ozdemir, E., Morsi, B.I., Schroeder, K.T., 2005. Sequestration of carbon dioxide in coal with enhanced coalbed methane recovery - A review. Energy and Fuels 19 (3), 659-724.

Wong, S., Law, D., Deng, X., Robinson, J., Kadatz, B., Gunter, W.D., Jianping, Y., Sanli, F., Zhiqiang, F., 2007. Enhanced coalbed methane and CO2 storage in anthracitic coals-Micro-pilot test at South Qinshui, Shanxi, 
China. International Journal of Greenhouse Gas Control 1 (2), 215-222.

Yates, D.J.C., 1954. The expansion of porous glass on the adsorption of non-polar gases. Proceedings of the Royal Society of London A 224 (1159), 526-544. 